\title{
Correction to: Integrated Safety Analysis of Abrocitinib for the Treatment of Moderate-to-Severe Atopic Dermatitis From the Phase II and Phase III Clinical Trial Program
}

\author{
Eric L. Simpson ${ }^{1}$. Jonathan I. Silverberg ${ }^{2} \cdot$ Audrey Nosbaum $^{3} \cdot K^{2}$ Nin L. Winthrop ${ }^{1} \cdot$ Emma Guttman-Yassky $^{4}$. \\ Karin M. Hoffmeister ${ }^{5,6} \cdot$ Alexander Egeberg $^{7} \cdot$ Hernan Valdez $^{8} \cdot$ Min Zhang $^{9} \cdot$ Saleem A. Farooqui ${ }^{10}$. \\ William Romero ${ }^{11} \cdot$ Andrew J. Thorpe $^{12} \cdot$ Ricardo Rojo $^{13} \cdot$ Susan Johnson ${ }^{12}$ (I)
}

Published online: 13 October 2021

(c) Springer Nature Switzerland AG 2021

\section{Correction to: \\ American Journal of Clinical Dermatology \\ (2021) 22:693-707 \\ https://doi.org/10.1007/s40257-021-00618-3}

The article was originally published electronically on SpringerLink on 18 August 2021. After publication in volume 22 , issue 5, pages 693-707, the authors highlighted that, in Supplemental Table 1, the overall patient numbers for the JADE MONO-2 and JADE COMPARE studies were incorrect. These errors have been rectified and a new corrected version of Supplementary file 1 has been uploaded with the manuscript on SpringerLink.

The original article has been corrected.
The original article can be found online at https://doi.org/10.1007/ s40257-021-00618-3.

Susan Johnson

Susan.L.Johnson@pfizer.com

1 Department of Dermatology, Oregon Health and Science University, Portland, OR, USA

2 Department of Dermatology, The George Washington University School of Medicine and Health Sciences, Washington, DC, USA

3 Department of Allergy and Clinical Immunology, Centre Hospitalier Lyon-Sud, Hospices Civils de Lyon, Pierre-Bénite, France

4 Department of Dermatology and Immunology, Icahn School of Medicine at Mount Sinai, New York, NY, USA

5 Versiti, Translational Glycomics Center, Blood Research Institute, Milwaukee, WI, USA
6 Departments of Biochemistry and Medicine, Medical College of Wisconsin, Milwaukee, WI, USA

7 Department of Dermatology, Bispebjerg Hospital, University of Copenhagen, Copenhagen, Denmark

8 Pfizer Inc., New York, NY, USA

9 Pfizer Inc., La Jolla, CA, USA

10 Pfizer R \& D UK Ltd., Kent, UK

11 Pfizer Ltd., Surrey, UK

12 Pfizer Inc., Collegeville, PA, USA

13 Pfizer Inc., Groton, CT, USA 